
approches théoriques et méthodologiques

\title{
Réflexions sur la prise en compte de l'évolution des concepts dans les systèmes d'organisation des connaissances
}

Reflections on Considerations of the Evolution of Concepts in Knowledge

Organization Systems

Nabil Ben Abdallah

\section{OpenEdition}

Journals

Édition électronique

URL : http://journals.openedition.org/edc/3886

DOl : 10.4000/edc.3886

ISSN : 2101-0366

Éditeur

Université Lille-3

Édition imprimée

Date de publication : 1 décembre 2012

Pagination : 117-139

ISBN : 978-2-917562-08-6

ISSN : 1270-6841

Référence électronique

Nabil Ben Abdallah, «Réflexions sur la prise en compte de l'évolution des concepts dans les systèmes d'organisation des connaissances », Études de communication [En ligne], 39 | 2012, mis en ligne le 01 décembre 2014, consulté le 30 avril 2019. URL : http://journals.openedition.org/edc/3886 ; DOI : 10.4000/edc.3886

Ce document a été généré automatiquement le 30 avril 2019.

(c) Tous droits réservés 


\title{
Réflexions sur la prise en compte de l'évolution des concepts dans les systèmes d'organisation des connaissances
}

\author{
Reflections on Considerations of the Evolution of Concepts in Knowledge \\ Organization Systems
}

Nabil Ben Abdallah

\section{Introduction}

1 Faciliter et assurer l'accès aux documents et à l'information constituent toujours la préoccupation majeure des spécialistes de l'information (bibliothécaires, documentalistes, archivistes, etc.). Pour atteindre cet objectif, des outils tels que les listes d'autorité, les thésaurus et les ontologies ${ }^{1}$ sont conçus et mis à la disposition des usagers et des utilisateurs. Ces outils sont particulièrement utiles pendant les étapes de repérage et de récupération des documents du processus de recherche d'information (Aytac et al., 2011 ; Greenberg, 2011 ; Perugini, 2010 ; Tennis et Sutton, 2008).

Dans une première acception, le thésaurus, l'ontologie ou le système d'organisation des connaissances d'une façon générale peuvent être décrits comme une collection de concepts. La manière d'organiser les concepts au sein d'un thésaurus, par exemple, peut être dictée par le souci d'avoir une classification la plus proche possible du domaine à décrire. Ce souci d'avoir une organisation des concepts reflétant la réalité à modéliser soulève, dans la plupart des cas, au moins deux critiques. La première critique tient à ce que l'organisation des concepts traduit souvent une vision unique de la réalité à modéliser, ce qui empêche toute autre représentation du domaine concerné. L'utilisateur $\mathrm{du}$ système d'organisation des connaissances est ainsi directement ou indirectement influencé par cette vision du domaine, un domaine qu'il tente d'explorer au travers des 
documents et des informations proposées par le système. La deuxième critique tient à ce que l'organisation des concepts ne prend généralement pas en compte l'évolution des concepts et des relations qui existent entre eux. Ce dernier point a un lien étroit avec la nature des concepts et soulève par conséquent une question d'ordre épistémologique sur le statut à accorder aux concepts représentés dans un système d'organisation des connaissances. Il est évident que les phénomènes qui sont à l'origine de ces deux critiques ne sont pas indépendants l'un de l'autre, ils sont corrélés. Par exemple, la multiplicité des points de vue dans un domaine donné peut-être une source de contradiction qui entraîne une évolution des concepts et des relations entre eux.

3 Nous commencerons pa $\mathrm{r}$ exposer un travail de Birger Hjørland sur les théories de concepts, en nous intéressant particulièrement aux différentes fonctions des concepts. Nous rappellerons ensuite que la théorie de l'activité est considérée comme une approche systémique proposant des unités d'analyse, des concepts et des principes utiles pour comprendre les éléments en jeu dans une activité humaine. Le modèle d'Engeström, en tant que forme de conceptualisation de la structure d'activité, sera employé pour analyser les éléments en interaction dans deux activités de recherche. Nous nous intéresserons en particulier aux processus sous-jacents à l'évolution des significations attribuées aux concepts.

\section{Idées et réflexions}

4 Dans un article récent, Hjørland (2009) affirme que la meilleure façon de comprendre les théories de concepts est de les classer selon des théories épistémologiques ${ }^{2}$ telles que l'empirisme, le rationalisme, l'historicisme, ou le pragmatisme. Chacune de ces épistémologies a ses propres théories de concepts. A partir de quelques exemples spécifiques de certaines disciplines, par exemple le concept d'« espèce » en biologie, Hjørland démontre que la façon, dont les chercheurs et les spécialistes définissent un concept est déterminée par leurs modèles théoriques. Nous suggérerons plus loin que d'autres aspects et facteurs sont à l'origine de la façon dont les communautés définissent un concept donné.

5 Hjørland pense que les théories de concepts les plus pertinentes sont celles qui sont reliées au pragmatisme et à l'historicisme. Son article commence par un constat sur la difficulté de définir ce qu'est un concept, quelles sont les théories de concepts les plus pertinentes et comment ces théories peuvent être classées. Hjørland cite Georges Rey qui, à partir d'une approche pragmatique, suppose que les concepts doivent être définis par rapport aux tâches que nous voulons qu'ils effectuent pour nous. Par conséquent, si nous ne pouvons pas définir les buts ou les objectifs de ces tâches, nous ne pouvons pas non plus définir les concepts. Rey met l'accent sur la difficulté de s'accorder sur les différentes fonctions des concepts. Il considère que ces fonctions peuvent être regroupées en quatre catégories principales : fonction de stabilité, fonction linguistique, fonction métaphysique et fonction épistémologique. Hjørland estime que la fonction de stabilité est probablement la principale fonction puisqu'elle influence et conditionne les autres. Il a cherché à déterminer et à expliquer les mécanismes régissant la fonction de stabilité des concepts. Pour lui, la meilleure explication de ces mécanismes est fournie par la théorie de l'activité, selon laquelle les concepts sont stabilisés par des activités ${ }^{3}$ standardisées au sein d'une communauté donnée. Les concepts sont ainsi considérés comme des outils élaborés pour réfléchir et communiquer à propos de ces pratiques 
collectives et sociales. Cette élaboration collective des concepts rompt avec une conception individuelle défendue par la psychologie développementale et les sciences cognitives.

6 Selon la théorie de l'activité, l'apprentissage de la signification des concepts (objets des activités) se réalise au travers de la compréhension de l'historique et de la dynamique des actions humaines sur ces objets. L'apprentissage d'un concept n'est pas seulement le fait d'associer des mots (signes) à des objets disposant de propriétés données (forme, couleur, matière, etc.) ou d'associer une entrée sensorielle donnée avec un ensemble de catégories logiques. Apprendre un concept, c'est aussi apprendre quelque chose sur la ou les fonctions du concept et les expériences sociales accumulées à propos de ces fonctions. Selon Vygotsky (1978), la langue est le moyen que nous employons pour internaliser notre expérience externe (nos actions sur les objets du monde).

7 Hjørland s'est basé sur la théorie de l'activité pour affirmer que :

Concepts are dynamically constructed and collectively negotiated meanings that classify the world according to interests and theories. Concepts and their development cannot be understood in isolation from the interests and theories that motivated their construction, and, in general, we should expect competing conceptions and concepts to be at play in all domains at all times $(2009,1522)$.

8 Cet extrait de l'article de Hjørland est une forme d'interprétation de la théorie de concepts résultant de la théorie de l'activité. L'affirmation que le développement des concepts ne peut être saisi indépendamment des intérêts et des théories qui ont motivé ce développement est en accord avec le principe de la structure hiérarchique admettant l'existence de trois niveaux pour mener une tâche donnée : les activités en relation étroite avec des motivations et des intentions, les actions subordonnées et liées à des buts spécifiques et les opérations déterminées par les conditions effectives de l'activité.

9 Nous pensons toutefois que l'article de Hjørland ne décrit pas clairement comment la théorie de l'activité peut être utilisée pour nous renseigner sur l'aspect dynamique et collectif des significations attribuées aux concepts. Qu'est-ce qu'une " pratique » humaine ? Quels sont les mécanismes sous-jacents à la création et à l'évolution des concepts au sein d'une communauté donnée ? Comment des opinions minoritaires au sein d'une communauté donnée peuvent-elles être à l'origine de développement des concepts alternatifs ? Voici des exemples de questions, en relation directe avec la construction et l'évolution des concepts, auxquelles la théorie de l'activité peut probablement apporter des éléments de réponse.

10 Dans la section suivante, nous décrivons brièvement les origines et les principes de la théorie de l'activité ainsi que les composants du système d'activité tels qu'ils sont définis par Engeström.

\section{Structure du système d'activité humaine}

11 La théorie de l'activité a été initiée par Vygotsky durant les années 1920 et au début des années 1930. Selon Vygotsky, les fonctions mentales supérieures, comme la pensée et la mémoire, doivent être considérées comme des produits d'une activité médiatisée dont le rôle de médiateur est attribué aux outils psychologiques et aux moyens de communication interpersonnelle. Cette idée est souvent schématisée par un triangle reliant le sujet, l'objet et l'outil (artefact) de médiation (Figure 1). Ce modèle en triangle ignore les relations sociales et n'étend pas l'analyse à d'autres sujets impliqués 
directement ou indirectement dans le déroulement de l'activité. En effet, l'analyse est focalisée sur le sujet dans sa quête motivée et médiatisée de l'objet ${ }^{4}$ (ici au sens de l'objectif de l'activité). Leontiev, disciple et collègue de Vygotsky, constate que la description initiale de l'activité telle qu'elle a été conceptualisée par ce dernier est insuffisante. Leontiev élargit les travaux initiaux sur la théorie de l'activité en séparant l'action individuelle de l'activité collective. Selon lui, l'activité dans un sens étroit (par rapport au plan psychologique de l'individu), représente une " entité de vie " médiatisée par la réflexion mentale. La fonction réelle de cette entité est d'orienter le sujet dans le monde des objets. Plus récemment, Engeström $(1987,2001)$ a complété le modèle de triangle (sujet, outil, objet) initialement élaboré par Vygotsky en y ajoutant l'élément communauté et deux éléments médiateurs : les règles et la division du travail. Pour Engeström, les activités humaines sont un phénomène collectif à la fois en ce qui concerne la participation (activités réalisées par des communautés) et en ce qui concerne la forme (activités réalisées collectivement). L'activité est observée comme un système avec sa propre structure, ses propres transformations internes et son propre développement.

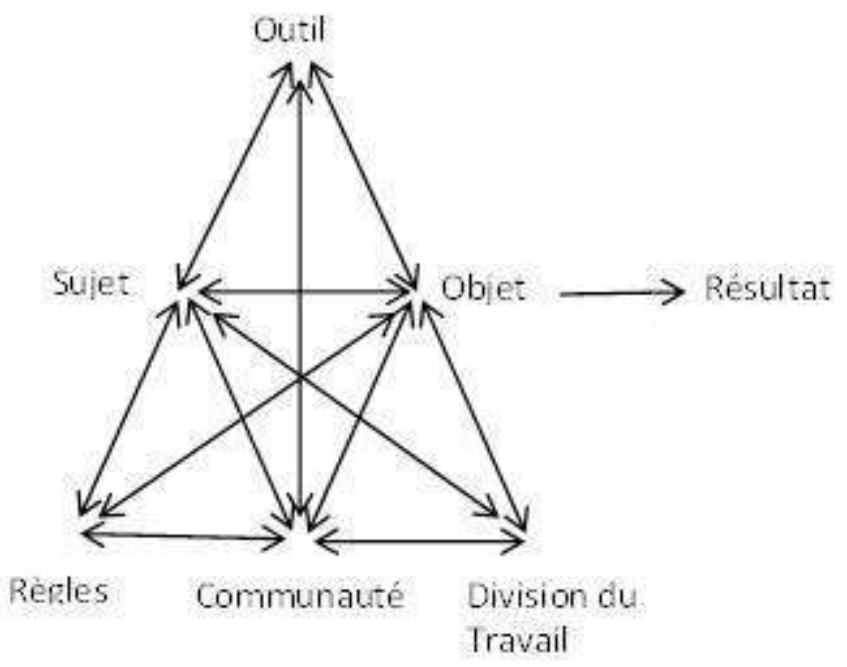

Figure 1 : Structure du système d'activités selon Engeström (1987)

Le système d'activité humaine d'Engeström est différent de celui de Leontiev. Pour le premier, le système d'activité est exclusivement collectif même si le sujet apparaît comme un composant du modèle (Figure 1). En effet, le sujet réel au sein du système d'activité est bien la communauté. Pour le second, les activités peuvent être individuelles ou collectives en ce qui concerne leur forme, mais il considère que l'objet d'activité est principalement un objet individuel. Cette focalisation sur l'individu n'empêche pas Leontiev de considérer que les activités sont toujours des activités sociales. Selon lui :

under whatever kind of conditions and forms human activity takes place, whatever kind of structure it assumes, it must not be considered as isolated from social relations, from the life of society. In all of its distinctness, the activity of the human individual represents a system included in the system of relationships of society (Leontiev, 1978, http://www.marxists.org/archive/leontev/works/1978/ch3.htm). Le triangle sujet-communauté-objet représente un processus reliant l'objet à une communauté de travail qui contribue, avec le sujet, à transformer l'objet en résultat. La 
relation entre le sujet et l'objet est médiatisée par l'outil et la communication entre la communauté et le sujet est médiatisée par des règles explicites ou tacites ; enfin, la relation entre la communauté et l'objet est médiatisée par une certaine forme de division $\mathrm{du}$ travail. L'ensemble constitue un système de relations dynamiques qui s'ajuste et s'adapte continuellement pour maintenir une certaine stabilité productrice (transformation de l'objet en résultat). Les principes couramment admis de la théorie de l'activité sont : le principe orienté-objet (object orientation), le principe de médiation, le principe de la structure hiérarchique d'activité, le principe d'internalisation/ externalisation et le principe de développement.

Selon le principe orienté objet de l'activité, toutes les activités humaines sont dirigées vers des objets. Elles permettent de transformer un objet considéré comme matière première en un objet significatif et potentiellement partagé par différents systèmes d'activité. Ces interactions avec l'objet sont, d'après le principe de médiation, forcément médiatisées par des outils matériels et/ou intellectuels. Ces outils peuvent disposer de la capacité de s'adapter à des environnements constitués d'autres outils et/ou d'autres acteurs humains. Le principe de la structure hiérarc hique est une des principales contributions de Leontiev (1981) qui admet l'existence de trois niveaux hiérarchiques pour mener une tâche donnée : les activités en relation étroite avec des motivations et des intentions, les actions subordonnées et liées à des buts spécifiques et les opérations déterminées par les conditions effectives de l'activité. Les niveaux sont interdépendants, chaque niveau est à la fois une condition au niveau supérieur et un contexte au niveau inférieur. L'échec ou la réussite à un niveau donné a des conséquences sur les deux autres ; ils peuvent perturber ou assurer le déroulement de l'activité. Pour Leontiev, l'activité humaine n'existe que sous forme d'action ou de chaîne d'actions ; il admet que :

if the actions that constitute activity are mentally subtracted from it, then absolutely nothing will be left of activity (Leontiev, 1978, http://www.marxists.org/ archive/leontev/works/1978/ch3.htm).

15 Le principe d'internalisation-externalisation nous renseigne sur les processus mentaux qui sont les conséquences des activités externes internalisées. Ces processus sont reliés à leur environnement social et culturel. Enfin, le principe de développement exige que l'activité humaine soit analysée dans le contexte de son développement. Il s'agit de comprendre comment une activité se développe, au fil du temps, dans un contexte historique et culturel, et comment les actions entreprises sur l'objet de l'activité affectent tel développement. Selon Kaptelinin et Bonnie :

principles of activity theory should be considered as an integrated system because they are associated with various aspects of the whole activity. That is, systematic application of any of the principles makes it eventually necessary to engage all the others $(2006,72)$.

La théorie de l'activité est aujourd'hui utilisée dans différents travaux qui s'intéressent directement ou indirectement aux activités humaines. Dans le champ du comportement informationnel (information behaviour) des sciences de l'information, quelques chercheurs (Spasser, 1999 et 2002 ; Wilson, 2006 et 2008 ; Xu, 2007 ; Allen et al., 2008 et 2011) ont tenté, à la lumière de la théorie de l'activité, de réexaminer les modèles existants des comportements informationnels pour proposer un cadre intégré expliquant, entre autres, les mécanismes gouvernant les interactions entre les utilisateurs et les systèmes de recherche d'information. Spasser pense que " la théorie de l'activité peut fournir à la science de l'information un vocabulaire riche, unifié et heuristiquement précieux, et un cadre conceptuel qui facilite à la fois l'amélioration continue de la pratique et assure la 
transférabilité et l'accumulation des connaissances » $(1999,1137)$. Toutefois, le peu de travaux utilisant la théorie de l'activité comme cadre théorique laisse penser que l'intérêt de cette théorie pour la recherche en sciences de l'information n'est pas encore bien établi. En nous basant sur la définition d'un système d'activité, nous considérons qu'une activité ne peut être réalisée que par des sujets motivés ; elle vise à transformer un objet en résultat. L'objet est partagé par les membres d'une communauté travaillant ensemble pour atteindre un objectif commun. Les outils, les règles et la division du travail médiatisent la relation entre le sujet, la communauté et l'objet.

\section{Comprendre le résultat d'une activité}

17 Nous essayons dans cette section d'illustrer notre propos sur l'aspect dynamique et collectif des significations attribuées aux concepts par un exemple de modélisation, selon le modèle d'Engeström, d'une activité de recherche dans le domaine du développement durable. Nous commençons par décrire une étude réalisée au sein d'un institut de recherche ${ }^{5}$ puis, en fonction des éléments d'information à notre disposition, nous déterminons les éléments constitutifs du système d'activité qui en résulte.

Des chercheurs de l'Institut de Recherche pour le Développement (IRD) sont partis de l'hypothèse que la production agricole est fortement affectée par le changement climatique, surtout dans les régions du monde qui ont connu des périodes de sécheresse. Pour plusieurs populations de ces régions, les céréales constituent la base habituelle de l'alimentation. Il est donc nécessaire, pour la sécurité alimentaire, de sauvegarder et de préserver la diversité de ces céréales. Avec des partenaires de l'IRD, les chercheurs ont étudié l'impact des changements sociaux (augmentation de la démographie et changement des modes de production) et environnementaux sur les variétés des céréales cultivées dans une région donnée où le défi majeur est de satisfaire les besoins de la population en dépit du changement climatique et de la raréfaction des terres cultivables. Pour les chercheurs, il est donc essentiel de comprendre comment les céréales cultivées réagissent aux changements climatiques.

Le travail des chercheurs consiste à faire une étude comparative d'échantillons de variétés de céréales locales prélevés en deux dates différentes et dans des localités couvrant l'ensemble de la région étudiée. L'étude a démontré qu'en dépit des changements climatiques, la diversité des variétés de céréales est maintenue. Elle révèle également une adaptation des variétés locales aux changements climatiques par le développement d'une floraison plus rapide et d'une diminution de la taille des épis. L'étude confirme que la production de céréales dans la région demeure possible même lorsque la saison pluvieuse est raccourcie. Ces résultats ont amené les chercheurs à s'intéresser aux processus génétiques de ces adaptations. Pour explorer ces processus, les chercheurs ont pris en compte divers facteurs, dont l'évolution des pratiques culturales. La sélection des semences par les paysans et les hybridations entre variétés sauvages et cultivées représentent des processus à saisir pour arriver à une gestion durable des ressources céréalières de la région étudiée.

20 Il est évident que les quelques informations à notre disposition sur le projet de recherche et le fonctionnement de l'Institut ne permettent pas de décrire en détail les actions individuelles et collectives effectuées lors de la réalisation du projet. Mais ces 
informations sont suffisantes pour analyser, en utilisant le modèle d'Engeström, le système d'activité résultant de cette activité de recherche.

21 La figure 2 décrit schématiquement la structure de l'activité de l'équipe ou l'unité de recherche (sujet) impliquée dans le projet décrit ci-dessus. Les chercheurs veulent comprendre comment les céréales cultivées dans la région réagissent aux changements climatiques (objet). Il s'agit d'une déclinaison particulière de l'objet « conséquences du changement climatique ", un objet relativement stable dans les activités de recherche de l'Institut. Ainsi, nous pouvons lire dans la rubrique « Orientations Stratégiques » du site de l'IRD : « Les grands enjeux de la recherche pour le développement vont se concentrer sur trois domaines interdépendants : les conséquences du changement climatique, les dynamiques démographiques et la mondialisation ». Les chercheurs disposent de leur savoir conceptuel et pratique d'un (outil) très important pour mener leur étude : les échantillons de variétés de céréales locales prélevés en deux dates différentes. La ( communauté) de l'Institut est large : elle regroupe plusieurs unités et laboratoires. Des règles et des principes sont établis par l'IRD afin de mener des projets de recherche pour le développement. Par exemple, dans le guide des bonnes pratiques de la recherche pour le développement publié par l'IRD, nous pouvons lire : « La protection des populations, des ressources naturelles et de l'environnement feront l'objet de dispositions spécifiques dans tout programme ou projet de recherche susceptibles d'interférer avec eux ». Le fonctionnement par projets de l'Institut nécessite sûrement une (division du travail) entre les chercheurs et leurs partenaires (universités, agences de recherche, organismes internationaux, etc.).

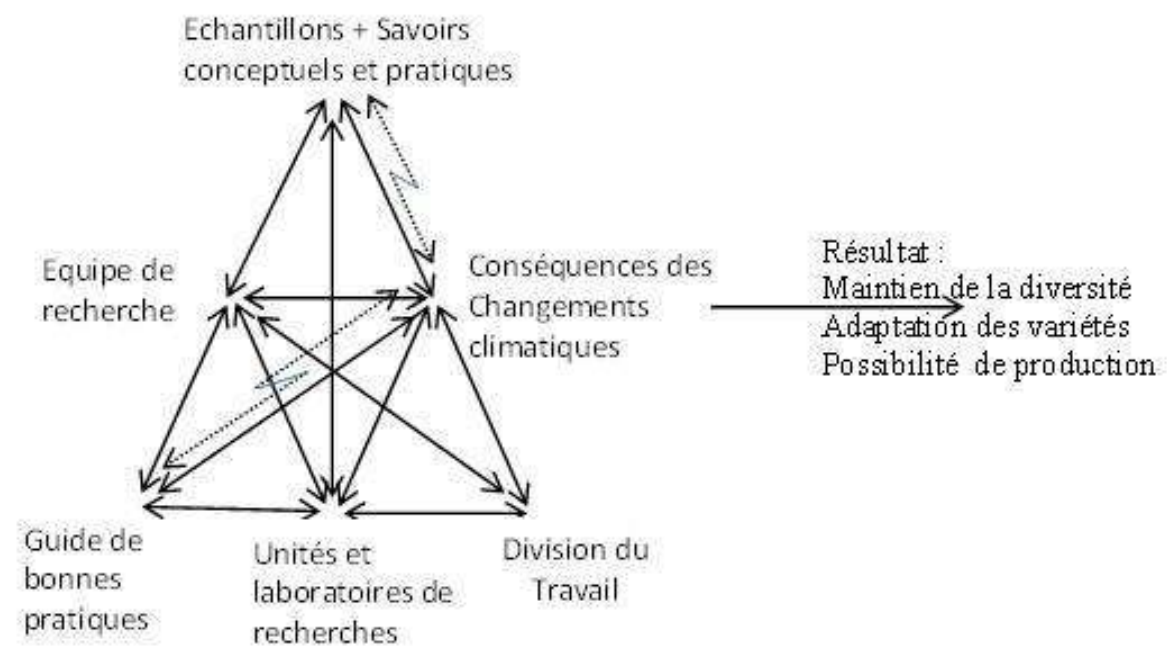

Figure 2 : Système d'activité d'une équipe de recherche lors de la réalisation d'un projet

Nous pouvons résumer ainsi les résultats de l'activité de recherche portant sur l'objet « adaptation des céréales aux évolutions climatiques " : (1) maintien de la diversité de céréales en dépit des changements climatiques; (2) adaptation des variétés locales aux changements climatiques (floraison plus rapide et diminution de la taille des épis); (3) production de céréales possible même lorsque la saison pluvieuse est raccourcie ; (4) résulte des points précédents : essayer de comprendre le processus génétique sousjacent en prenant en compte divers facteurs, dont l'évolution des pratiques culturales. 
Quel est l'apport de l'analyse de cette activité de recherche à notre réflexion sur l'aspect dynamique et collectif des significations attribuées aux concepts? Nous apprenons de cette analyse que les chercheurs impliqués dans le projet utilisent, entre autres, une approche comparative pour étudier des échantillons soigneusement constitués selon des méthodes standardisées. Le savoir conceptuel et pratique des chercheurs et les règles établies par l'Institut (par exemple, le respect d'une démarche qualité) garantissent l'obtention de résultats valides. Entre les chercheurs impliqués dans le projet et les autres chercheurs de l'Institut, il existe nécessairement une certaine forme d'entente ne seraitce que sur l'objet de l'activité. Dans un tel contexte, nous ne nous attendons pas à un changement majeur dans les significations associées aux concepts utilisés comme " changement climatique ", " sécurité alimentaire ", etc. Toutefois, quelques résultats du système d'activité peuvent amener les chercheurs à poser des questions sur les significations apparemment stabilisées de quelques concepts ou proposer des candidats concepts pour décrire et expliquer un phénomène nouveau. Dans notre exemple, la tentative des chercheurs de comprendre les processus génétiques sous-jacents à l'adaptation des céréales au changement climatique pourrait les amener, par exemple, à une acception différente du phénomène de "floraison " ou le développement de nouveaux concepts pour expliquer des aspects inconnus de ces processus. La ou les théories utilisées par le sujet (équipe de recherche) pour interagir avec l'objet de l'activité n'expliquent pas à elles seules les significations attribuées aux différents concepts employés comme outil ou étudiés comme objet. Ces significations sont déterminées par l'ensemble des éléments constitutifs du système d'activité. Ainsi, par exemple, la signification attribuée par les chercheurs au concept « changement climatique » n'est pas déterminée seulement par les théories qu'ils emploient mais aussi par leurs actions sur des déclinaisons de l'objet " conséquences du changement climatique ». Ces actions sont régies par des règles de production scientifique imposées par l'Institut, par la communauté avec laquelle les chercheurs de l'équipe collaborent, par la division du travail qui se met en place en fonction de l'objet de l'activité, et enfin par les outils matériels et/ou conceptuels utilisés. Dans notre exemple, le résultat de l'activité de recherche portant sur l'objet " effet du changement climatique sur la culture des céréales" nous permet d'établir des relations sémantiques entre : changement climatique, floraison des céréales, processus d'adaptation, culture des céréales et taille des épis. Ces relations nous apprennent que le changement climatique influence la floraison des céréales et la taille des épis, déclenche un processus d'adaptation, et n'empêche pas, contrairement à ce qui est attendu, la culture des céréales (Figure 3). 


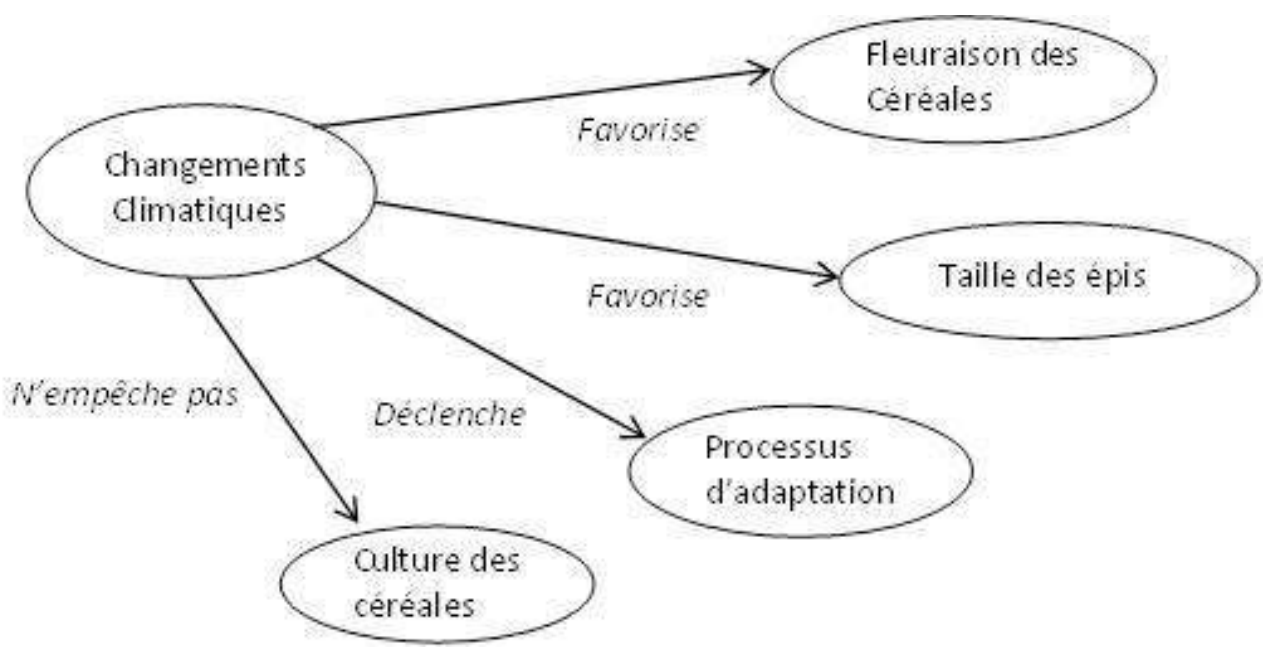

Figure 3 : Relations sémantiques déduites de l'activité deux rôles différents du concept au sein d'un système d'activité, ceux de médiateur et
d'objet. En effet, les concepts (généralement introduits par la théorie employée) peuvent être utilisés par le sujet et la communauté comme un outil qui médiatise ainsi leurs interactions avec l'objet de l'activité. Les concepts peuvent être aussi l'objet sur lequel portent les actions du sujet et de la communauté. La modification de la signification des concepts « objet " peut être déterminée à partir du résultat du système d'activité. En revanche, la modification de la signification des concepts "outil» ne peut être déterminée qu'à partir des contradictions qui émergent, par exemple, entre l'objet et l'outil ou entre l'objet et les règles. Dans la figure 2, les contradictions sont représentées par des flèches en forme d'éclair. L'outil se révèle insuffisant pour expliquer un comportement non prévu de l'objet. Dans une telle situation, le processus d'évolution des concepts est généralement trop long et ne peut être saisi à partir d'un seul système d'activité. Les mêmes contradictions doivent être observées dans différents systèmes d'activité pour qu'il y ait nécessité de faire évoluer ces concepts.

Notre analyse n'a porté que sur un seul système d'activité, soit une activité relative à la réalisation d'un projet. Or nous avons plusieurs systèmes d'activité en interaction l'un avec l'autre. D'autres équipes ou unités de recherche travaillent sur des recherches dont l'objet peut être une autre déclinaison de l'objet « conséquences du changement climatique » ou une déclinaison d'autres objets de recherche de l'Institut : « dynamiques démographiques » et " mondialisation ». L'analyse de plusieurs systèmes d'activité nous permet de mieux cerner la modification des significations attribuées aux concepts " objet » et aussi de mieux définir les objets dont les limites et les fonctions ne sont pas encore bien définies. Par exemple, l'analyse de plusieurs systèmes d'activité dont les objets sont des déclinaisons de l'objet "mondialisation " fournit de précieux enseignements sur le champ sémantique de la mondialisation.

En conclusion de ce premier exemple d'analyse d'une activité de recherche selon le modèle d'Engeström, nous estimons que plusieurs éléments du processus d'attribution des significations aux concepts ont été déterminés. Notre analyse confirme que la définition des concepts et des relations sémantiques peut être déterminée à partir de l'étude des systèmes d'activité qui impliquent la construction, l'évolution et l'utilisation 
de ces concepts. L'étude doit concerner à la fois les résultats (par exemple la littérature produite) et les éléments constitutifs de ces systèmes. Nous apportons ici, peut-être, une réponse à la question posée par Hjørland :

Thus, a basic problem in KO is whether semantic relations are a priori or a posteriori: whether they can be known before examining the literature or only after such an examination has been carried out. What kind of literary warrant (or other kind of warrant) is needed in order to identify semantic relations and classify concepts? $(2007,378)$.

\section{Comprendre l'objet d'une activité}

Dans l'exemple précédent, nous avons vu que le résultat d'un système d'activité dépend de l'objet de l'activité mais aussi des autres éléments constitutifs du système tels que le sujet, la communauté, l'outil, les règles et la division du travail. Toutefois, nous avons tendance à penser que l'objet reste le déterminant principal du résultat d'un système d'activité et que les autres éléments constitutifs du système n'ont qu'un rôle secondaire dans la détermination du résultat. Afin de mesurer l'importance de ces éléments constitutifs, nous analysons rapidement dans la présente section des systèmes d'activité différents mais orientés vers le même objet. Nos exemples sont extraits d'une étude explorant les fondements théoriques des sciences de l'information.

L' étude est rapportée dans quatre articles : Zins (2007b) présente 130 définitions des concepts " donnée ", " information » et " connaissance ", rédigées par 45 chercheurs en sciences de l'information de différents pays. Zins (2007a) fournit 50 définitions des sciences de l'information et décrit six conceptions différentes du champ de l'information. Zins (2007d) présente une collection de 28 schémas de classification décrivant des aspects différents des sciences de l'information au début du XXI siècle. Zins (2007c) expose une carte de connaissances globale et systématique basée sur dix catégories principales : fondement, ressources, travailleurs du savoir, contenu, applications, opérations et processus, technologies, environnements, organisations et utilisateurs. Nous nous basons ici essentiellement sur le troisième article (Zins, 2007d), qui présente des schémas de classification.

Zins (l'enquêteur) demande à chaque membre du panel d'élaborer un schéma de classification représentant sa conception des sciences de l'information. Chaque schéma doit être exhaustif et représenter les caractéristiques principales de la discipline. Il doit être systématique, les catégories de chaque niveau étant mutuellement exclusives et collectivement exhaustives. Chaque membre du panel révise à deux reprises sa proposition de schéma, après lecture et commentaires des autres chercheurs.

Dans ce qui suit nous analysons, toujours selon le modèle d'Engeström, le systë fme d'activité résultant du traitement par les chercheurs de la question de l'enquêteur. La figure 4 décrit schématiquement la structure de l'activité d'un chercheur (sujet) traitant la question de l'enquêteur. Le chercheur doit analyser et étudier les caractéristiques et les phénomènes explorés par les sciences de l'information (objet) afin de concevoir un schéma de classification représentant sa conception de cette science. Le chercheur confirmé dans sa discipline connaît certainement plusieurs approches théoriques et différents schémas de classification et il les utilise comme (outil) pour élaborer son propre schéma. Le chercheur semble travailler seul mais en réalité il appartient à une ( communauté) qui peut être constituée de ses collègues à l'université ou des membres de 
son équipe de recherche. En outre, dans notre exemple, le chercheur est influencé directement ou indirectement par les propositions des autres membres du panel étant donné qu'il peut être amené à réviser sa proposition en fonction de leurs schémas. En effet, le protocole de l'étude crée une sorte de communauté virtuelle des membres du panel. Le cadre de l'étude impose des (règles) que le chercheur doit respecter. Ainsi, on exige des membres du panel que leurs schémas soient adéquats, exhaustifs, systématiques et basés sur des fondements théoriques et philosophiques solides. En plus des règles imposées par le cadre général de l'étude, les membres du panel sont susceptibles d'être amenés à respecter aussi les règles imposées par leurs communautés respectives, par exemple celle de mettre en valeur les résultats des recherches de leurs communautés. La ( division du travail) ne semble pas être nécessaire dans l'activité que nous sommes en train d'analyser. Cependant, le fait que chaque membre du panel a des liens avec au moins une communauté, permet de croire qu'une forme de division du travail peut être utile pour interagir avec l'objet de l'activité. Enfin, le (résultat) attendu de cette activité portant sur l'objet " sciences de l'information » doit être un schéma de classification (quel qu'en soit le type : taxinomie, typologie, ou ontologie) adéquat, exhaustif, et systématique.

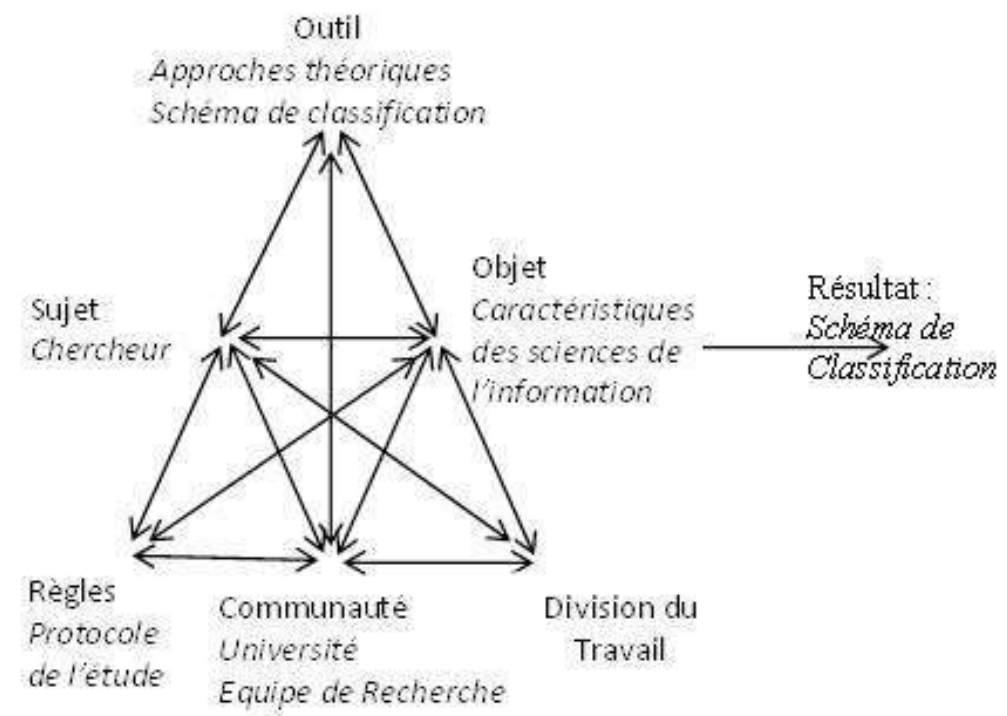

Figure 4 : Système d'activité d'un chercheur lors du traitement de la demande de l'enquêteur

$$
\text { proposés représentent des conceptions différentes des sciences de l'information. Voici }
$$
comment Zins explique ces conceptions différentes :

It is assumed that every one of the 28 schemes demonstrates a distinct conception of IS. For example, if one asserts that IS explores all the aspects of the data, information, knowledge, message (D-I-K-M) phenomena, I would expect his or her scheme to include all the aspects of the D-I-K-M phenomena... However, the vast majority of the panel adopts the culture model ${ }^{6}$. Evidently, the culture model represents the mainstream of contemporary Information Science (2007d, 668).

Le point de vue de l'auteur et les commentaires des membres du panel permettent de penser que l'approche théorique adoptée par chaque chercheur est à l'origine de sa conception des sciences de l'information (objet de l'activité), et donc de son schéma de classification. Nous retrouvons d'une certaine manière le point de vue de Hjørland : « It 
is, thus, demonstrated that the way scholars define a given concept is a reflection of their methodological ideals » $(2009,1520)$.

A notre avis, cette explication est insuffisante ; elle ignore d'autres aspects qui sont à l'origine des différentes conceptions proposées par les chercheurs. Le choix par un chercheur d'une approche théorique donnée n'est probablement pas aléatoire ; plusieurs raisons motivent son choix, il maîtrise bien l'approche choisie et il est convaincu qu'elle est la seule qui lui permet de répondre correctement à la question posée. En outre, ses interactions avec les membres de sa communauté et les règles imposées par le cadre général de l'étude sont probablement aussi des éléments déterminants non seulement le choix de l'approche théorique à adopter, mais aussi la manière selon laquelle cette approche est interprétée et utilisée. Les schémas de classification proposés semblent être des propositions des chercheurs individuels, mais ils sont en réalité des propositions socialement construites. Les chercheurs n'ignorent pas nécessairement l'utilité de leurs schémas, ce qui peut considérablement influencer leurs propositions. La fonction utilité de la conception n'est certainement pas loin de l'esprit des chercheurs au moment de la rédaction de leurs propositions. Rey, cité par Hjørland (2009), souligne le principe pragmatique qui veut que les concepts soient définis en relation avec le travail que nous voulons qu'ils réalisent pour nous. Par conséquent, si nous ne pouvons préciser nos objectifs et nos attentes, nous ne pouvons pas préciser nos concepts.

Dans notre exemple, les schémas de classification représentent des acceptions différentes de l'objet (concept) « sciences de l'information ». Les membres du panel ont tous interagi avec le même objet mais dans des systèmes d'activité différents. Les outils, les règles, la communauté et les motivations ne sont pas les mêmes d'un système d'activité à un autre, ce qui explique clairement les significations différentes attribués au même objet. Pour un système d'organisation des connaissances, il n'est ni possible ni utile de faire une synthèse des différents schémas, leur nombre et leur contenu ne pouvant être déterminés a priori. A notre avis, la validité d'un schéma donné ne peut être mesurée que par rapport à son utilité en tant qu'outil de différents systèmes d'activité. C'est une manière d'éviter un arbitraire qui pourrait être assimilé à une certaine forme de pragmatisme.

\section{Conclusion}

Hjørland affirme que les significations des concepts sont dynamiquement construites et collectivement négociées. Il met ainsi l'accent sur l'aspect social de la construction des concepts et considère qu'ils sont des outils élaborés par la société (les communautés) pour réfléchir et communiquer sur ses activités. Les idées de Hjørland sont évidemment inspirées de la théorie de l'activité qui considère que notre conscience du monde qui nous entoure est formée par nos actions sur ses objets et, réciproquement, notre conception (ou représentation) interne du monde est fixée par notre activité dans le monde. Les objets (par exemple les concepts) focalisent l'attention, la motivation et l'effort de ceux qui, à travers leurs activités, modifient et créent de nouveaux objets. Les nouveaux objets ne sont pas souvent des produits intentionnels d'une simple activité mais plutôt des conséquences inattendues de multiples activités. Pour Engeström (2009), la pertinence sociétale et l'impact de la théorie de l'activité dépend de notre capacité à saisir le caractère changeant des objets (conceptuels et matériels) sur lesquels portent nos activités. C'est cet te facette dynamique et collective des concepts (objets) que nous avons essayé de traiter dans cet article. 
L'analyse de deux exemples de systèmes d'activité nous a permis de conclure que : (1) la ou les théories utilisées par le sujet pour interagir avec l'objet de l'activité n'expliquent pas à elles seules les significations attribuées aux différents concepts employés comme outil ou étudiés comme objet ; ces significations sont déterminées par l'ensemble des éléments constitutifs du système d'activité ; (2) les concepts ont deux rôles distincts au sein d'un système d'activité : ils peuvent être utilisés par le sujet et la communauté comme outil médiatisant les interactions avec l'objet, et ils peuvent aussi être l'objet sur lequel portent des actions ; (3) la modification de la signification des concepts « objet " est déterminée à partir du résultat du système d'activité, mais la modification de la signification des concepts "outil " n'est observée qu'à partir des contradictions qui émergent, par exemple, entre l'objet et l'outil ou entre l'objet et les règles ; (4) l'analyse de plusieurs systèmes d'activité partageant le même objet, ou plus précisément les déclinaisons d'un même objet, nous permet de mieux cerner la modification des significations attribuées à ces objets et aussi de mieux définir les objets dont les limites et les fonctions ne sont pas encore bien définies (par exemple le réchauffement climatique, l'e-gouvernement, l'e-démocratie, etc.).

Les réflexions esquissées ici doivent être comprises comme des hypothèses de travail destinées à mieux comprendre l'aspect dynamique et collectif des significations attribuées aux concepts. Il s'agit d'une tentative d'atteindre à une compréhension globale d'un phénomène dont dépend la qualité des systèmes d'organisation des connaissances.

\section{BIBLIOGRAPHIE}

Allen D. K., Wilson T. D., Norman A. W. T. et Knight C., (2008), Information on the move : the use of mobile information systems by UK police forces, Information Research, vol. 13, $\mathrm{n}^{\circ} 4$.

Allen D., Karanasios S. et Slavova M., (2011), Working with activity theory : Context, technology, and information behaviour, Journal of the American Society for Information Science and Technology, vol. $62, \mathrm{n}^{\circ} 4$, pp. $776-788$.

Aytac S., Kipp M. E., Neal D., Rubin V. L., Pattuelli C. et Hsieh-Yee I., (2011), Emerging trends in knowledge organization and information organization course curriculum, in Proceedings of the American Society for Information Science and Technology, vol. 48, $\mathrm{n}^{\circ}$ 1, pp. 1-4.

Engeström Y., (1987), Learning by Expanding : An Activity-Theoretical Approach to Developmental Research, Helsinki, Finland, Orienta-Kosultit Oy.

Engeström Y., (2001), Expansive Learning at Work : toward an activity theoretical reconceptualization, Journal of Education and Work, vol. 14, $\mathrm{n}^{\circ}$ 1, pp. 133-156.

Engeström Y., (2009), The Future of Activity Theory : A Rough Draft, in Sannino A., Daniels H. et Gutiérrez K. D., Learning and Expanding with Activity Theory, Cambridge University Press. 303-328.

Greenberg J., Losee R., Pérez Agüera J. R., Scherle R., White H. and Willis C., (2011), HIVE : Helping Interdisciplinary Vocabulary Engineering, Bulletin of the American Society for Information Science and Technology, 37 (4), 23-26. 
Hjørland B., (2007), Semantics and knowledge organization, Annual Review of Information Science and Technology, vol. 41, $\mathrm{n}^{\circ}$ 1, pp. 367-405.

Hjørland B., (2009), Concept theory, Journal of the American Society for Information Science and Technology, vol. 60, nº 8, pp. 1519-1536.

Kaptelinin V. et Nardi B. A., (2006), Acting with technology : Activity Theory And Interaction Design, Cambridge, Mass., MIT Press.

Leontiev A., (1978), Activity, Consciousness, and Personality, http://www.marxists.org/archive/ leontev/works/1978/index.htm.

Leontiev A., (1981), Problems of the Development of the Mind, Moscow, Progress.

Perugini S., (2010), Supporting multiple paths to objects in information hierarchies : Faceted classification, faceted search, and symbolic links, Information Processing \& Management, vol. 46, $\mathrm{n}$ - 1, pp. 22-43.

Spasser M. A., (1999), Informing information science : the case for activity theory, Journal of the American Society for Information Science, vol. 50, $\mathrm{n}^{\circ} 12$, pp. 1136-1138.

Spasser M. A., (2002), Realist activity theory for digital library evaluation: Conceptual framework and case study, Computer Supported Cooperative Work (CSCW), vol. 11, n 1, pp. 81-110.

Tennis J. T. et Sutton S. A., (2008), Extending the simple knowledge organization system for concept management in vocabulary development applications, Journal of the American Society for Information Science and Technology, vol. 59, n 1, pp. 25-37.

Vygotsky L. S., (1978), Mind in society : The development of higher psychological processes, Harvard University Press, Cambridge, MA, USA.

Wilson T. D., (2006), A re-examination of information seeking behavior in the context of Activity Theory, Information Research, vol. $11, \mathrm{n}^{\circ} 4$.

Wilson T. D., (2008), Activity theory and information seeking, Annual Review of Information Science and Technology, vol. 42, $\mathrm{n}^{\circ} 1$, pp. 119-161.

Xu Y., (2007), The dynamics of interactive information retrieval behavior, Part I : An activity theory perspective, Journal of the American Society for Information Science and Technology, vol. $58, \mathrm{n}$ - 7, pp. 958-970.

Zins C., (2007a), Conceptions of information science, Journal of the American Society for Information Science and Technology, vol. 58, $n^{\circ} 3$, pp. 335-350.

Zins C., (2007b), Conceptual approaches for defining data, information, and knowledge, Journal of the American Society for Information Science and Technology, vol. 58, $\mathrm{n}^{\circ}$ 4, pp. 479-493.

Zins C., (2007c), Knowledge map of information science, Journal of the American Society for Information Science and Technology, vol. 58, $\mathrm{n}^{\circ}$ 4, pp. 526-535.

Zins C., (2007d), Classification schemes of Information Science : Twenty-eight scholars map the field, Journal of the American Society for Information Science and Technology, vol. 58, $\mathrm{n}^{\circ} 5$, pp. 645-672.

\section{NOTES}

1. En plus de représenter, à la manière d'un thésaurus, les relations entre des termes connexes, l'ontologie offre la possibilité de décrire explicitement ces relations, ce qui permet d'enrichir de 
façon considérable la structure sémantique caractérisant le modèle du domaine étudié. Cet enrichissement sémantique permet, sous certaines conditions, de faire des inférences à partir de l'ontologie.

2. Dans la tradition anglo-saxonne, l'épistémologie ne s'intéresse pas seulement aux connaissances scientifiques, elle est jointe à l'idée de théorie de la connaissance.

3. Hjørland utilise le terme " pratiques".

4. Il est important de signaler ici que l'objet d'une activité dénote à la fois l'aspect objectif (adjectif) de l'activité portant sur des objets matériels ou intellectuels qui existent indépendamment du sujet et l'aspect subjectif (objet en tant que but) de l'activité qui dépend des besoins et des motivations du sujet.

5. Nous nous sommes très largement inspirés d'une recherche réalisée au sein de l'Institut de Recherche pour le Développement (IRD) pour construire notre exemple. La description fournie dans notre article ne reprend donc pas tous les éléments d'information publiés sur le site de l'IRD (www.ird.fr) concernant cette recherche.

6. "Information science is the study of the mediating aspects of D-I-K-M phenomena as they are implemented in the cultural (i.e., social) domain » (Zins, 2007a, 340).

\section{RÉSUMÉS}

Le présent article utilise la recherche de Hjørland (2009) comme point de départ pour réfléchir à l'aspect dynamique et collectif des significations attribuées aux concepts. Le modèle d'Engeström est utilisé pour analyser deux activités de recherche afin de saisir les processus sous-jacents à la création et à l'évolution des concepts au sein d'une communauté donnée. Le résultat de l'analyse montre que les théories utilisées par les chercheurs pour interagir avec l'objet de l'activité n'expliquent pas à elles seules les significations attribuées aux différents concepts employés comme « outil » ou étudiés comme « objet ». Ces significations sont déterminées par l'ensemble des éléments constitutifs du système d'activité. Cet article est une tentative de démontrer et d'expliquer l'utilité de la théorie de l'activité dans l'étude de l'organisation des connaissances.

This paper uses Hjørland's research (2009) as a starting point for pondering the dynamic and collective meanings attributed to concepts. Engeström's model is used to analyze two research activities in order to understand the processes underlying the creation and evolution of concepts within a given community. The results of the analysis show that the theories used by researchers to interact with the object of the activity do not by themselves explain the meanings attributed to the different concepts used as tools or studied as objects. These meanings are determined by all of the components of the activity system. This paper is an attempt at demonstrating and explaining the utility of activity theory in the study of knowledge organization.

\section{INDEX}

Mots-clés : signification des concepts, théorie de l'activité, rôle des concepts, système d'organisation des connaissances, système d'activité

Keywords : meanings of concepts, activity theory, role of concepts, knowledge organization systems, activity systems 
AUTEUR

NABIL BEN ABDALLAH

Nabil Ben Abdallah - ELICO - Équipe de Recherche de Lyon en Information et en Communication 